\title{
Manifestation of optical vortices on the surface of solids under irradiation with femtosecond laser pulses
}

\author{
N.G. Zubrilin, I.M. Dmitruk \\ Institute of Physics, NAS of Ukraine \\ 46, prospect Nauky, 03680 Kyiv, Ukraine, \\ Phone: 38 (044)525-16-70,e-mail: zubrilin@iop.kiev.ua
}

\begin{abstract}
Quasi-periodic microstructures containing dislocations are formed on the surfaces of metals and semiconductors under irradiation with high-power femtosecond laser pulses. Interpretation of microstructures as a result of interference of the incident plane wave and surface waves leads to the logical conclusion about the relationship of dislocations in the interference fringes with optical vortices in surface wave.
\end{abstract}

Keywords: laser processing, surface wave, optical vortex.

Manuscript received 21.01.14; revised version received 23.04.14; accepted for publication 12.06.14; published online 30.06.14.

\section{Introduction}

It is known [1-5] that under the action of plane-polarized laser pulses with a wavelength $\lambda$ on the surface of metals and some semiconductors, structures in the form of parallel strips with a period $\Lambda$, somewhat smaller than the wavelength $\lambda$, oriented perpendicular to the plane of the electric vector of light, are observed.

There are several possible mechanisms of formation of these structures. Proposed in [1] is an explanation of the effect based on the concept of interference of the incident and scattered along the surface electromagnetic waves. There is also a hypothesis about the nature of these structures as frozen capillary waves on the surface of the molten by laser layer [6]. However, the most probable mechanism of formation of these structures is associated with interference of the incident and surface electromagnetic waves excited by the incident wave on the surface of metal at their scattering by roughness of the metal surface [7].

In this paper, we report on the important feature of laser-induced structures on the surface of metal, which, as far as we know, hasn't been still given proper attention.

\section{Experimental methods}

The experiment was carried out at Femtosecond Laser Center for collective use at the Institute of Physics, NAS of Ukraine, by irradiating the metal surface with the Tisapphire femtosecond laser system consisting of a master oscillator Mira-900F and a regenerative amplifier Legend HE. The laser pulse with the wavelength of $820 \mathrm{~nm}$, duration of $140 \mathrm{fs}$, and energy of about 0.8 to $1 \mathrm{~mJ}$ was focused on the surface with a long focal length lens. The spot size on the surface was $0.8 \ldots 1 \mathrm{~mm}$, which corresponds to the power density of the order of $10^{12}$ up to $10^{13} \mathrm{~W} / \mathrm{cm}^{2}$, the time of irradiation was $0.4 \ldots 2 \mathrm{~s}$. If desired, a larger surface area was treated, while the sample was moved during irradiation at a constant velocity of several millimeters per second. Thus, every part of the surface was treated with hundreds to thousands of laser pulses. Formation of quasi-periodic structures on the surface was monitored visually by appearance of bright light diffraction on the treated areas.

The detailed study of morphology inherent to the treated surface was performed with a scanning electron microscope JEOL JXA-8200. The typical image of the irradiated surface of tungsten is shown in Fig. 1. There is 
a quasi-periodic system of bands with the period $\sim 0.5 \mu \mathrm{m}$, which remains constant throughout the treated area.

However, you may notice that sometimes the band breaks (indicated by arrows in Fig. 1) with the corresponding deformation of the bands around, while the distance between the bands remains unchanged. Thus, dislocations occur in the system of bands. As noted above, the obtained system of bands on the surface is most logically explained as the result of interference of the incident and surface waves (surface plasmon polariton in the case of metals). In this case, disappearance of the interference fringe indicates the change of the phase difference of the interfering waves by $2 \pi$ when going around this point, i.e. the presence of the screw dislocation of the wavefront of one of the waves - the optical vortex. These dislocations in the interference pattern with vortex beams are well known [8].

Similar structures were obtained on metals (W, Mo, $\mathrm{Ta}, \mathrm{Ni}, \mathrm{Ag}, \mathrm{Pt})$. Preliminary experiments showed that these structures are observed also on the surface of semiconductors ( $\mathrm{Si}$ for example).

Noteworthy is that in the stripes and between them spherical shape nanostructures with the size $\sim 30 \ldots 100 \mathrm{~nm}$ are present (Fig. 2).

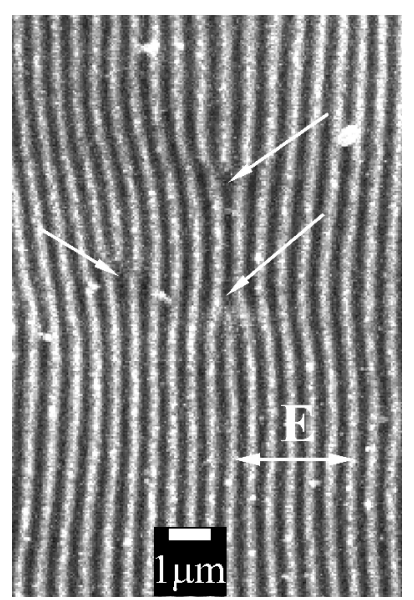

Fig. 1. Characteristic "forks" associated with the presence of "optical vortices" in electromagnetic fields taking part in formation of structures on the surface of tungsten.

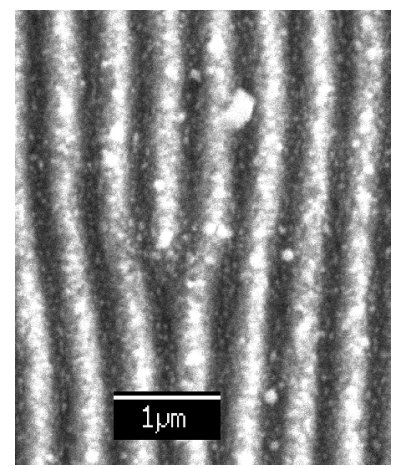

Fig. 2. Image of treated tungsten surface with larger magnification reveals nanostructures in the stripes and between them.

\section{Results and discussion}

The beams with helical wavefront perturbations (singular beams) occupy a special place among the wave beams with a variable structure of the amplitude-phase profile. This kind of disturbance causes the vortex nature of the propagating light energy, which suggests the existence of peculiar optical vortices [9].

To generate singular optical beams, most widely used are the methods for direct conversion of the spatially inhomogeneous phase of the light wave and holographic interferometry methods of converting light beams. In the first case, spiral phase plates or liquid crystal spatial modulators are used. Another method to generate singular beams is diffraction of the initial Gaussian beam on special optical transparencies based on computer-generated holograms [9].

Finally, we discuss briefly a possible mechanism of formation of optical vortices when the metal surface is irradiated by power femtosecond pulses.

Under "natural conditions", most of the wavefront dislocations of laser beams that cause the vortex structure of the light field are observed in the scattering of light by random media on the propagation path (for example, when passing sufficiently large tract in the atmosphere or scattering on very rough surfaces).

It is known that during irradiation of rough surfaces with laser, speckles appear. In [10], it is pointed out for the first time that the wavefront dislocations are inherent to optical fields that have a speckle structure. For monochromatic polarized beam with sufficiently developed inhomogeneities of complex amplitude, appearance of wavefront dislocations is inevitable [10].

When discussing the possible mechanism of formation of optical vortices as a result of power laser pulse interaction with the metal surface, note the similarity of the resulting fringe pattern on the metal surface with the transparencies used to produce beams with vortices. Besides, as formation of the structures on the surface occurs gradually, there takes place an accumulation effect of sequential exposure from hundreds or thousands of pulses. The surface of metal may be not only a recording medium, but it can also participate in formation of spatial distribution of the amplitude and phase of the wavefront, i.e. operate as a space-phase modulator. We can assume that initially small surface roughnesses are minor points forming wavefront dislocations, which then as a result of interference with the incident plane wave form a quasiperiodic structure with dislocation that in turn acts as a phase modulator and so on. Thus, a positive feedback in the system is realized as a result of formation of a distinct stable self-consistent structure under the action of laser pulses.

\section{Conclusion}

The study of periodic structures formed on the surface of metals and semiconductors under irradiation with 
femtosecond laser pulses showed characteristic quasiperiodic patterns with dislocations (broken stripes). The offered interpretation associates these dislocations in interference pattern with the presence of wavefront dislocations - optical vortices on the surface or scattered waves. Most distinct structures were observed on the surface of tungsten, which is probably due to its high melting point.

\section{Acknowledgements}

The authors thank V.B. Sobolev (Technical Center NAS of Ukraine) for carrying out electron microscopy of the samples. Partial financial support came from Ukrainian State target scientific and technical program "Nanotechnologies and Nanomaterials" for 2010-2014 project No. 1.1.3.11, 3.5.5.23.

\section{References}

1. D.C. Emmony, R.P. Howson, and L.J. Willis, Laser mirror damage in germanium at $10.6 \mu \mathrm{m} / / \mathrm{Appl}$. Phys. Lett. 23, p. 598-600 (1973).

2. Zhou Guosheng, P.M. Fauchet, and A.E. Siegman, Growth of spontaneous periodic surface structures on solids during laser illumination// Phys. Rev. B, 26(10), p. 5366-5381 (1982).

3. J.F. Young, J.S. Preston, H.M. van Driel, and J.E. Sipe, Laser-induced periodic surface structure. II. Experiments on $\mathrm{Ge}, \mathrm{Si}, \mathrm{Al}$, and brass // Phys. Rev. B, 27(2), p. 1155-1172 (1983).
4. Kiminori Okamuro, Masaki Hashida, Yasuhiro Miyasaka, Yoshinobu Ikuta, Shigeki Tokita, and Shuji Sakabe, Laser fluence dependence of periodic grating structures formed on metal surfaces under femtosecond laser pulse irradiation // Phys. Rev. B, 82, p. 165417-5 (2010).

5. Litao Qi, Kazuhiro Nishii, and Yoshiharu Namba, Regular subwavelength surface structures induced by femtosecond laser pulses on stainless steel // Opt. Lett. 34(12), p. 1846-1848 (2009).

6. T. Sano, M. Yanai, E. Ohmura, Y. Nomura, I. Miyamoto, A. Hirose, K. Kobayashi, Femtosecond laser fabrication of microspike-arrays on tungsten surface // Appl. Surf. Sci. 247, p. 340-346 (2005).

7. S.R.J. Brueck and D.J. Ehrlich, Stimulated surfaceplasma-wave scattering and growth of a periodic structure in laser-photodeposited metal films // Phys. Rev. Lett. 48(24), p. 1678-1681 (1982).

8. L.A. Kazak, A.L. Tolstik, Formation of superposition and stability of vortex optical beams of different orders // Vestnik Belorusskogo Gosudarstv. Universiteta, Ser. 1, №2, p. 3-7 (2010), in Russian.

9. M. Padgett, J. Courtial, L. Allen, Light's orbital angular momentum // Physics Today, 57(5), p. 3540 (2004).

10. N.B. Baranova, B.J. Zeldovich, A.B. Mamaev, N.F. Pilipetskii, V.V. Shkunov, Wavefront dislocations of speckle-inhomogeneous field (theory and experiment) // JETP Lett. 33(4), p. 206210 (1981), in Russian. 